EPJ Web of Conferences 73, 02020 (2014)

DOI: $10.1051 /$ epjconf/20147302020

(C) Owned by the authors, published by EDP Sciences, 2014

\title{
PDF studies at LHCb
}

Simone Bifani ${ }^{\mathrm{a}}$, on behalf of the LHCb Collaboration

University of Birmingham, School of Physics and Astronomy, Birmingham, UK

\begin{abstract}
Measurements of the $\mathrm{Z}$ and $\mathrm{W}$ boson production cross-sections provide important tests of the Standard Model at the LHC energies and allow the Parton Density Functions of the proton to be constrained. Electroweak bosons are reconstructed in several leptonic decay channels, and their cross-sections are reported using data collected in the forward region by the LHCb experiment at a centre of mass energy of $\sqrt{s}=7 \mathrm{TeV}$ with an integrated luminosity of up to $\sim 1 \mathrm{fb}^{-1}$. Results are compared to NNLO predictions.
\end{abstract}

\section{Introduction}

The LHCb [1] experiment, one of the four main detectors at the CERN Large Hadron Collider (LHC), is a single arm spectrometer fully instrumented in the forward region between pseudo-rapidities, $\eta$, of 2 and 5. Although primarily designed to study heavy flavour physics, the forward reach can provide important precision tests of the Standard Model at the LHC energies in a kinematic range which is complementary to the General Purpose Detectors. NNLO cross-section predictions have relative errors of between a few percents and $\sim 10 \%$, depending on rapidity, where the dominant uncertainty is due to the knowledge of the proton Parton Density Functions (PDFs). Since production in the forward region involves the interaction of one valence quark carrying a large fraction of the proton momentum (high- $x$ ) and a sea quark with a small fraction of the momentum (low- $x$ ), PDFs can be probed in two distinct regions of Bjorken $x$, one of which is previously unexplored.

The reported measurements are performed using data collected by the LHCb experiment at a centre of mass energy of $\sqrt{s}=7 \mathrm{TeV}$ during 2010 and 2011, corresponding to integrated luminosities of $37 \mathrm{pb}^{-1}$ and $1 \mathrm{fb}^{-1}$ respectively. $Z^{1}$ and $W$ boson production cross-sections are determined by reconstructing decays with final states containing leptons.

\section{2. $Z \rightarrow \mu \mu$}

$Z \rightarrow \mu \mu$ events are selected from the full 2011 dataset [2], by requiring two reconstructed muons with opposite charge, a transverse momentum, $p_{T}$, greater than $20 \mathrm{GeV} / \mathrm{c}$, lying in the pseudo-rapidity range between 2.0 and 4.5 and having a combined invariant mass in the range $60<M_{\mu \mu}<120 \mathrm{GeV} / \mathrm{c}^{2}$. Background events due to heavy flavour hadrons that decay semi-leptonically and QCD events where

\footnotetext{
${ }^{a}$ e-mail: simone.bifani@cern.ch

${ }^{1}$ Includes both the $Z$ and the virtual photon $\left(\gamma^{*}\right)$ contributions.
}

This is an Open Access article distributed under the terms of the Creative Commons Attribution License 4.0, which permits unrestricted use, distribution, and reproduction in any medium, provided the original work is properly cited. 

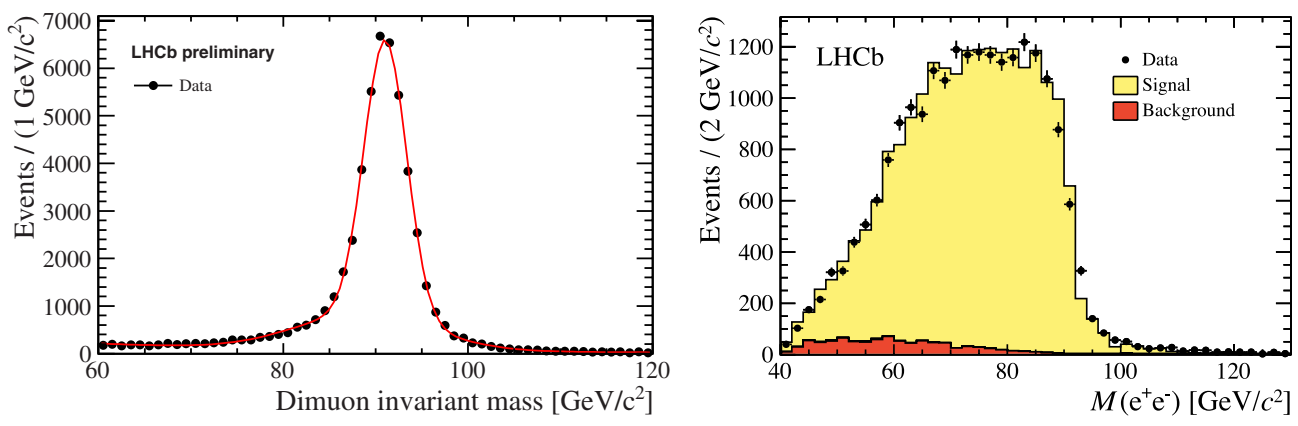

Figure 1. Di-lepton invariant mass distribution for the $Z \rightarrow \mu \mu$ (left) and $Z \rightarrow e e$ (right) final states.

kaons or pions either decay in flight or punch-through the detector to be falsely identified as muons are studied using data-driven methods. $Z \rightarrow \tau \tau$, top and di-boson productions are estimated using simulation. The selection retains 52626 candidate events, with a purity of $99.7 \%$. The di-muon invariant mass distribution is shown in Fig. 1 (left).

\section{$2.1 Z \rightarrow \mu \mu+j e t$}

Jet production in association with a $Z$ boson is studied on 2011 data using the $Z \rightarrow \mu \mu$ analysis described above as baseline selection [3]. Jets are reconstructed in the pseudo-rapidity range between 2 and 4.5 by using an anti- $k_{T}$ clustering algorithm with a radius parameter, $R$, of 0.5 . Each jet is required to have a transverse momentum above $10 \mathrm{GeV} / \mathrm{c}$ and to be well separated from the muons of the $Z$ decay. The jet energy is calibrated using simulation and cross-checked in data, and the corresponding resolution is $10-15 \%$ for jet transverse momenta between 10 and $100 \mathrm{GeV} / \mathrm{c}$. The fraction of $Z \rightarrow \mu \mu$ events with at least one jet is determined to be $22.9 \%$ with a background contamination which is consistent with the inclusive $Z$ analysis.

\section{3. $Z \rightarrow e e$}

$Z \rightarrow e e$ events are identified on the 2011 datasets [4]. Candidates are reconstructed by requiring two identified and oppositely charged electrons with a $p_{T}$ greater than $20 \mathrm{GeV} / \mathrm{c}$ and a pseudo-rapidity in the range $2.0<\eta<4.5$. Electron momentum, rather than energy, is used to reconstruct the two-body invariant mass, $M_{e e}$, which is required to be above $40 \mathrm{GeV} / \mathrm{c}^{2}$. Due to saturation of the electromagnetic calorimeter and an incomplete Bremsstrahlung recovery, the peak is significantly broader than in the di-muon final state. A sample of same-sign ee pairs provides a data-based estimate of background due to particle misidentification. Background from heavy flavour hadrons and $Z \rightarrow \tau \tau$ is found to be negligible. In total, 21420 candidates are selected with a purity of $95.5 \%$. Figure 1 (right) presents the di-electron invariant mass distribution.

\section{4. $Z \rightarrow \tau \tau$}

Data collected in 2011 are analysed to select $Z \rightarrow \tau \tau$ events using three leptonic and two semileptonic final states $(\mu \mu, \mu e, e \mu, \mu h$ and $e h)$ [5]. The leading lepton must have a transverse momentum above $20 \mathrm{GeV} / \mathrm{c}$, while the second particle is required to have a $p_{T}$ exceeding $10 \mathrm{GeV} / \mathrm{c}$. Leptons are required to have a pseudo-rapidity between 2.0 and 4.5, while hadrons are selected to be in the range 

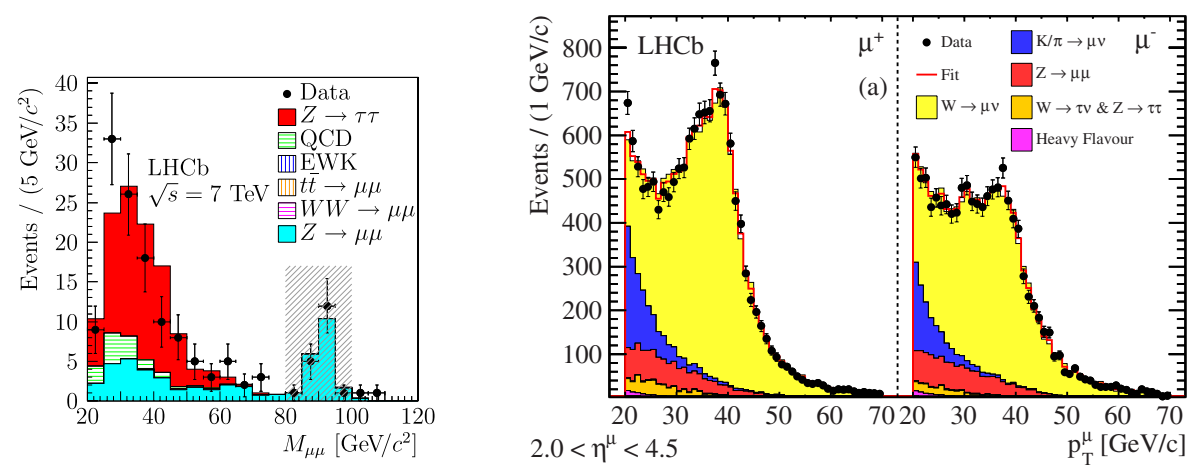

Figure 2. Di-muon invariant mass distribution for the $Z \rightarrow \tau \tau \rightarrow \mu \mu v_{\mu} v_{\mu} v_{\tau} v_{\tau}$ final state (left). Muon transverse momentum distribution of $W \rightarrow \mu v_{\mu}$ candidate events (right).

$2.25<\eta<3.75$. The invariant mass of the visible particles has to be greater than $20 \mathrm{GeV} / \mathrm{c}^{2}$. In order to reduce the background contamination, further requirements are made: particles must be isolated and back-to-back in the transverse plane; tracks are required to be inconsistent with production at the primary vertex for the $\mu \mu, \mu h$ and $e h$ cases; due to a larger background from Drell-Yan events in the $\mu \mu$ final state, tracks are required not to be balanced in $p_{T}$. A total of 990 events are selected with a purity that varies between $60 \%$ and $70 \%$ depending on the final state. The di-muon invariant mass distribution is shown in Fig. 2 (left).

\section{5. $\boldsymbol{W} \rightarrow \mu \boldsymbol{v}_{\mu}$}

$W$ candidates are selected in the $W \rightarrow \mu v_{\mu}$ channel from the full 2010 dataset [6] by requiring an isolated muon with $p_{T}>20 \mathrm{GeV} / \mathrm{c}$ and $2.0<\eta<4.5$. Due to the neutrino not being reconstructed, additional criteria are imposed on consistency with the primary vertex and event activity to further reduce the background contamination. The signal purity is estimated by fitting the lepton $p_{T}$ spectrum to the expected shapes for signal (simulation) and backgrounds (simulation and data) in 5 bins of the muon pseudo-rapidity. Background sources include $Z \rightarrow \mu \mu$ where one of the muons goes outside the LHCb acceptance, $W \rightarrow \tau \nu_{\tau}$ and $Z \rightarrow \tau \tau$ where one tau decays leptonically inside the detector to a muon, semi-leptonic decays of heavy flavour hadrons, and QCD events where kaons or pions are misidentified as muons due to decay in flight. Figure 2 (right) shows the fit result. A total of $14660 \mathrm{~W}^{+}$and $11618 \mathrm{~W}^{-}$events are identified, with a purity of $78.8 \%$ and $78.4 \%$ respectively.

\section{Results}

Production cross-sections are measured with the kinematic requirements that leptons have a transverse momentum greater than $20 \mathrm{GeV} / \mathrm{c}$, a pseudo-rapidity between 2.0 and 4.5 , and, in the case of the Z, a combined invariant mass between 60 and $120 \mathrm{GeV} / \mathrm{c}^{2}$. Results are corrected for acceptance, trigger, track finding, particle identification and selection efficiencies, and Final State Radiation (FSR). While the acceptance and FSR corrections are determined from simulation, efficiencies are primarily estimated using data-driven methods.

A summary of the measured $Z$ inclusive cross-sections is presented in Fig. 3 (left). The jet multiplicity distribution normalised to the total inclusive $Z$ cross-section is shown in Fig. 3 (right). The differential cross-section as a function of the $Z$ boson rapidity and the lepton charge asymmetry 

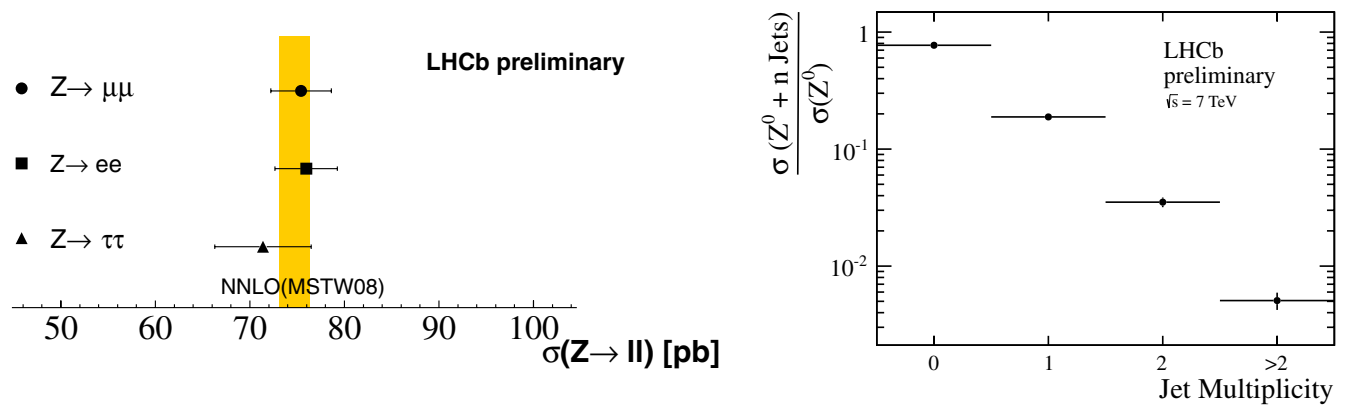

Figure 3. Inclusive $Z$ cross-section measurements compared to theoretical prediction (left) and jet multiplicity distribution in $Z \rightarrow \mu \mu$ events (right).
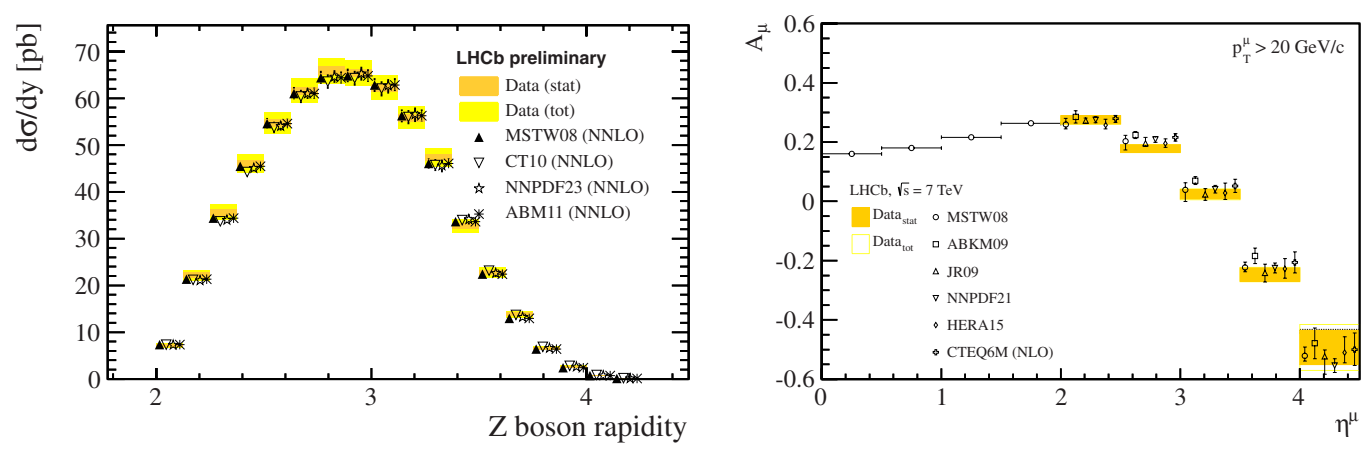

Figure 4. Differential cross-section for $Z$ production in bins of boson rapidity (left) and $W$ charge asymmetry in bins of lepton pseudo-rapidity (right) compared to theoretical predictions.

as a function of the muon pseudo-rapidity in case of the $W$ are shown in Fig. 4. All determinations are consistent with theoretical predictions calculated at NNLO using the DYNNLO [7] and FEWZ [8] generators with a number of different PDF sets.

\section{References}

[1] R. Aaij et al. [LHCb Collaboration], JINST 3, S08005 (2008)

[2] R. Aaij et al. [LHCb Collaboration], LHCb-CONF-2013-007

[3] R. Aaij et al. [LHCb Collaboration], LHCb-CONF-2012-016

[4] R. Aaij et al. [LHCb Collaboration], JHEP 1302, 106 (2013)

[5] R. Aaij et al. [LHCb Collaboration], JHEP 1301, 111 (2013)

[6] R. Aaij et al. [LHCb Collaboration], JHEP 1206, 058 (2012)

[7] S. Catani et al., Phys. Rev. Lett. 98, 222002 (2007)

[8] R. Gavin et al., Comput. Phys. Commun. 182, 2388 (2011) 